\title{
The introduction of AOI in PCB defect detection based on linear array camera
}

\author{
Min Guo 1,2,3,4 Rui Wang 1,2,3,4,a \\ ${ }^{1}$ College of Electronic Information \& Control Engineering, Beijing University of Technology, Beijing \\ 100124, China \\ ${ }^{2}$ Beijing Key Laboratory of Computational Intelligence and Intelligent System, Beijing 100124 , \\ China \\ ${ }^{3}$ Engineering Research Center of Digital Community, Ministry of Education, Beijing 100124, China \\ ${ }^{4}$ Beijing Laboratory for Urban Mass Transit, Beijing 100124, China \\ wangrui122499@sina.com
}

Keywords: AOI; camera calibration; PCB; defect detection

Abstract. Along with the rapid development of computer technology and digital image processing, traditional PCB technology of defect detection could not meet the demand of the quality of PCB, meanwhile AOI has received increasing attention. This paper mainly introduces the AOI machine based on PCB defect detection, hardware structure and software system is respectively carried on the design. In addition, provide a new method of defect detection which is based on the comparison of contour and introduce the process of camera calibration chiefly.

\section{Introduction}

With the development of science and technology, PCB (Printed Circuit Board) keep forward moving to the trend of high accuracy, high complexity and high performance, which challenge the quality of PCB detection ${ }^{[1]}$. Because of immature technology, high costs, low efficiency and other factors, traditional PCB defect detection method is hard to meet the demand of accuracy of PCB now, however, as a new automatic detection technology, AOI is just able to solve the problem. This paper studied the application of high-precision, high accuracy, enabling rapid detected AOI in PCB defect detection, which using a new method to detect PCB defection based on comparison of contour. Meanwhile, the camera calibration is also an important part of AOI.

\section{AOI Brief Introduction}

AOI system first analyzes and deals with standard PCB image information in CAM file. Then optical imaging unit is settled on a precision X-Y table, scanning the PCB which is to be detected ${ }^{[2]}$. It forms test PCB image in the computer. By comparing the standard image and test PCB image in logic, we can detect and classify the defect through its contour feature ${ }^{[3]}$. The last step is to make the data feedback to users. Hardware and software collaborative work and make a whole. AOI flow chart is shown in Fig.1.

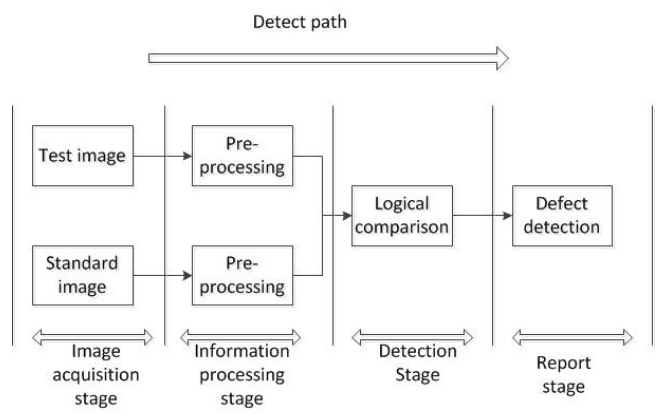

Fig.1. AOI flow chart 


\section{Hardware Structure}

\section{Hardware Introduction}

The hardware device used in this paper is CMOS black and white linear array camera, Germany AD4 silicon software acquisition card (Camera Link acquisition card) combined with opto trigger board, which is used to array trigger signal wiring. Optical source uses a custom linear combination of coaxial light, which form is coaxial optical source and multiple linear array optical source combination. The hardware structure of the system is shown in Fig.2.

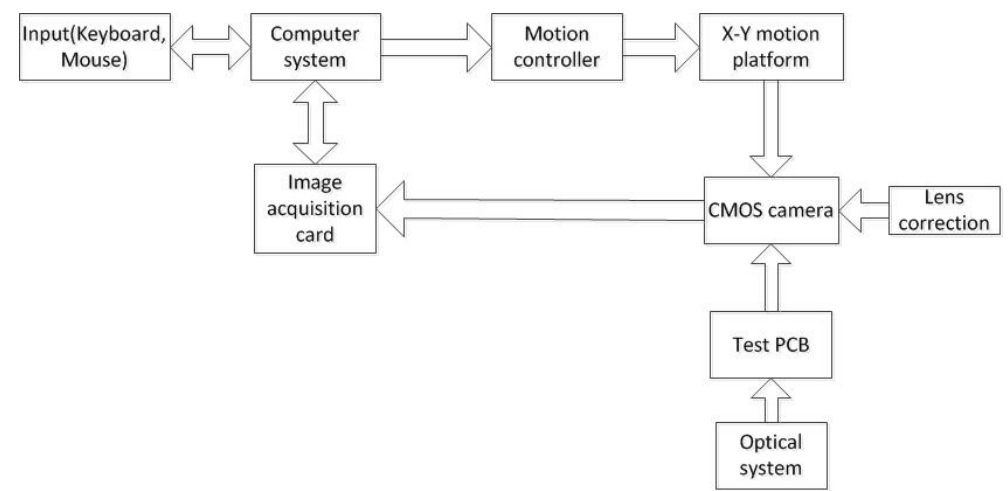

\section{Camera Calibration}

Fig. 2 hardware structure diagram

Because of the difference between real image and Ideal pinhole image, image distortion will occur when the camera capture images ${ }^{[4]}$. If you want to measure the actual length of an object accurately, you have to do camera calibration. Linear array camera calibration is about how to project the object in a three dimensional coordinate system to a two-dimensional plane. Linear array camera imaging model is shown in Fig.3.

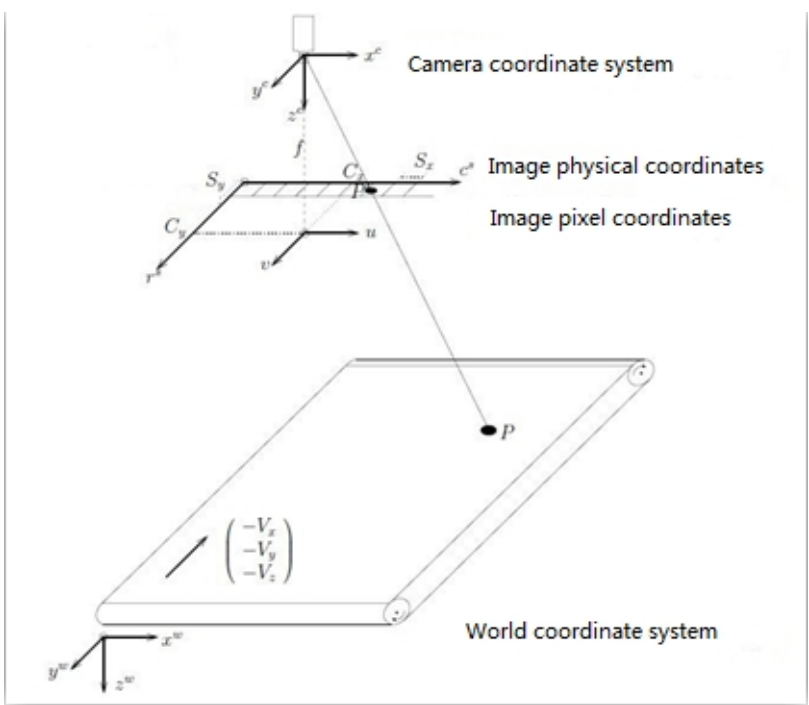

Fig.3 Line camera imaging model

There are four related coordinate system in the Calibration process ${ }^{[5]}$. They are world coordinate system, camera coordinate system, image physical coordinates system and image pixel coordinates system. The goal of camera calibration is the conversion relationship between image pixel coordinate system and world coordinate system. Camera calibration parameters obtained divided into external and internal parameters. External parameters are the conversion relationship between world coordinate system and camera coordinate system. Internal parameters are the conversion relationship between image pixel coordinate system and camera coordinate system. Parameters obtained from camera calibration determine the projection relationship from three-dimensional to two-dimensional. 
Only having a relative movement with the target object can shoot a useful image by linear array camera. The most common relative motion is the trajectory of the object relative to the camera along a straight line. In this situation, the relative movement can be represented as a three-dimensional vector $\mathrm{v}$. There is relative movement between the target object and the camera in the process of image acquisition. As a result, there is a relative movement between camera coordinate system and the target object. The position is different when shooting each row of pixels, each row in the image corresponds to a different position. In order to make the model easy to analyze, we can use known conditions that the movement between camera and target object is linear. We just need to know the conversion relationship between world coordinate system and camera coordinate system when shooting first row of pixels, then the position of each line can be struck by a three-dimensional vector $\mathrm{v}$.

\section{Software Section Introduction}

\section{Software Introduction}

The software design of PCB automatic detection system based on image processing is also very important ${ }^{[6]}$. The software design of AOI system completes the function of image data acquisition, image data processing and image data analysis unit, which including several digital image processing technology, such as image preprocessing, defect detection and recognition, etc. Meanwhile, it dose data exchange with operator. The system software flow chart is shown in Fig.4.

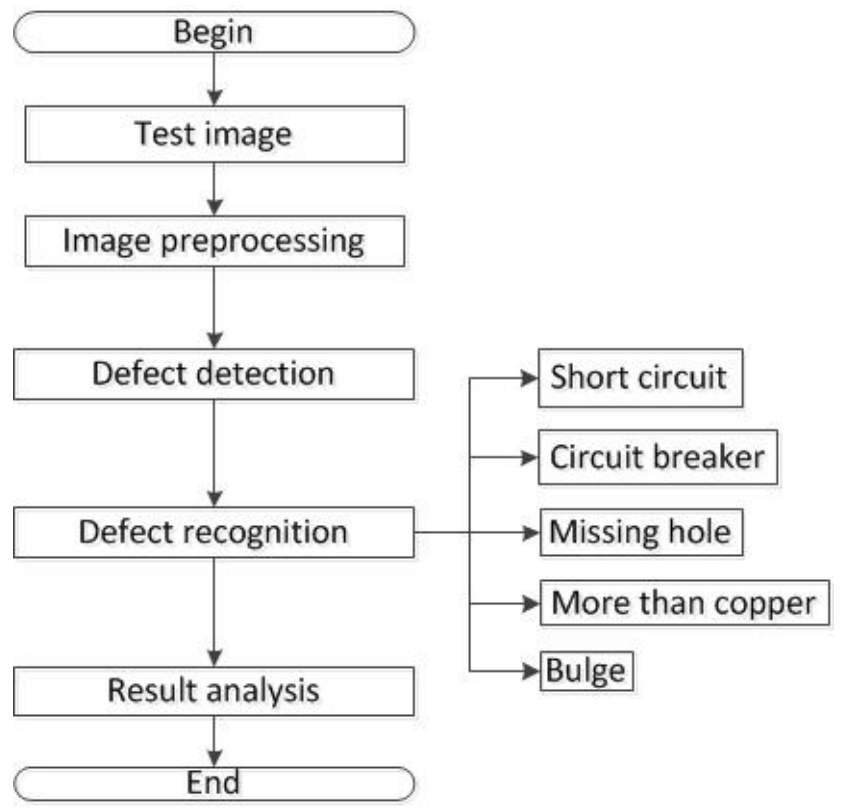

Fig.4 The system software flow chart

\section{Defect Detection}

Current PCB defect detection method is mainly divided into three categories: reference comparison, the non-reference comparison and mixing ${ }^{[7]}$. Because the pattern to be detected is complex, reference comparison method is the only option. This article does not use traditional reference comparison method based on content, however, uses a new method based on contour comparison. Firstly, extract the edge features of the test PCB image and standard image; secondly, compare and analysis the contour between test PCB image and standard image; thirdly, it will form a residual image. Compare the residual image .If the comparison result is white residual image (less contour pixels) or black residual image (more contour pixels), it shows that there are differences between test PCB image and standard image. It can be judged that the existence of defects in the PCB.

The specific steps of reference comparison method based on contour are as follows:

(1) Acquire test PCB image; 
(2) Image preprocessing, including trimming, median filter;

(3) Image location, which is aligned the position of test PCB image with the position of standard image;

(4) Defect detection, which uses edge contours comparison. Firstly, extract the edge information of template (usually Gerber file); secondly, extract the edge information of test PCB image. Edge information includes lines, circles and other characteristic attribute: length, focus, angle, fit, etc. Thirdly, compare the difference between test PCB image and standard image.

\section{Conclusions}

The results of this research are as follows:

First, study the application of AOI in PCB defect detection based on machine vision and digital image processing.

Second, introduce the method of line array camera calibration.

Third, propose a new method of PCB defect detection by comparison of contour.

Against the problem of backward technology in PCB defect detection, this paper provides a perfect solution. The system has accurate and rapid detection process, easy operation mode and provides a nice platform for PCB defect detection accurately and efficiently. This paper has important theory and application value. Due to time pressures, there are many aspects of the AOI system have not been studied, waiting for us to discover. It is believed that AOI will have a better and more comprehensive development in the field of PCB defect detection.

\section{Acknowledgements}

This work was financially supported by National Natural Science Foundation of China (61174109).

\section{References}

[1] Xiaoting Sun. The study of PCB visual inspection system [D]. Changsha: Central South University Master's thesis, 2008. (In Chinese)

[2] Yasuhiko hara, A System for PCB Automated Inspection Using Fluorescent Light, IEEE Transaction Analysis and Machine Intelligence, 1998,10(1): 101-104.

[3] A. P. Sprague, A Method for Automatic Inspection of Printed Circuit Boards, Image Understanding.2001,54(3):401-415.

[4] Hai Liu, Ye Li, Yanzhen Li. Design and Research Image Processing PCB automatic detection system [J] based electronic world, 2013 (4): 115-117. (In Chinese )

[5] Benhabib B, Charette C R, Smith K C, et al. Automatic visual inspection of printed circuit boards: An experimental system. Int. J. Robot. Autom., 1990, 5(2): 1034-1044

[6] Yingxin Wu. AOI effective applications in PCB inspection process [J] Printed Circuit Information, 2009 (S1): 382-390. (In Chinese)

[7] Rongqiong Huang. AOI undetected effective prevention programs in PCB applications [J] Printed Circuit Information, 2011 (6): 60-65. (In Chinese ) 